\title{
Percutaneous Ethanol Injection of Hepatic Tumors: Single-Session Therapy with General Anesthesia
}

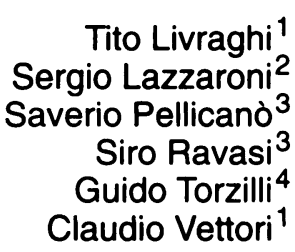

Received April 16, 1993; accepted after revision June 18, 1993.

1Divisione di Radiologia, Ospedale Civile, 20059 Vimercate, Milan, Italy. Address correspondence to T. Livraghi.

${ }^{2}$ Divisione di Medicina, Ospedale S. Biagio, 24023 Clusone, Bergamo, Italy.

${ }^{3}$ Divisione di Anestesia e Rianimazione, Ospedale Civile, 20059 Vimercate, Milan, Italy.

${ }^{4}$ Divisione di Chirurgia, Ospedale di Lodi, 20075 Lodi, Italy.

0361-803X/93/1615-1065

(C) American Roentgen Ray Society
OBJECTIVE. We studied the feasibility and the effectiveness of percutaneous ethanol injection, performed with general anesthesia in a single session, for treating malignant hepatic lesions.

SUBJECTS AND METHODS. We treated 30 patients with sonographically guided percutaneous injection of ethanol. Twenty had hepatocellular carcinoma and cirrhosis, and 10 had hepatic metastases, principally from colon cancer. The mean volume of ethanol injected was $57 \mathrm{ml}$ (range, 6-165 $\mathrm{ml}$ ).

RESULTS. CT showed complete necrosis (up to $8.2 \mathrm{~cm}$ ) in seven of 10 patients with encapsulated hepatocellular carcinoma and about $90 \%$ necrosis in the remaining three patients. In four of these patients, the $\alpha$-fetoprotein level fell from more than 200 $\mathrm{ng} / \mathrm{ml}$ to less than $20 \mathrm{ng} / \mathrm{ml}$ during treatment. In 10 patients with infiltrating hepatocellular carcinoma, about $70-90 \%$ necrosis was achieved; in six of these patients, the $\alpha$ fetoprotein level, which had been more than $200 \mathrm{ng} / \mathrm{ml}$, decreased during treatment. In the 10 patients with metastases, more than $50 \%$ necrosis was always achieved. Levels of carcinoembryonic antigen decreased after treatment in all patients. In three patients who had cirrhosis with superficial hepatocellular carcinoma, peritoneal hemorrhage occurred but did not require transfusion.

CONCLUSION. Our results show that percutaneous injection of ethanol in a single session with general anesthesia is feasible and effective and has several advantages over multisession therapy. These include shorter treatment time and the ability to treat larger and more numerous lesions.

\section{AJR 1993;161:1065-1069}

Percutaneous ethanol injection (PEI) under sonographic guidance has been shown to improve the survival of patients with hepatocellular carcinoma (HCC) and cirrhosis whose tumors are less than $5 \mathrm{~cm}$ in diameter [1-5]. The effectiveness of PEI in treating hepatic metastases has yet to be proved [6]. Treatment is usually given in an outpatient setting, twice weekly in three to 15 sessions, depending on the number of lesions and their size, in doses of only a few milliliters in order to avoid severe pain. For treatment with PEI, most researchers select patients with no more than three lesions, each with a diameter less than $3-5 \mathrm{~cm}$. To see whether PEI is feasible for treating patients with larger and more numerous lesions, even for palliative treatment, we conducted a feasibility trial of PEI in a single session with general anesthesia.

\section{Subjects and Methods}

\section{Subjects}

Thirty patients were treated, 20 patients with HCC and cirrhosis and 10 patients with hepatic metastases.

The patients with HCC included 16 men and four women from 32 to 75 years old (mean, 65 years old). Nineteen patients had disease classified as Child's class $A$ and one had class $B$ disease. Patients were selected because they could not have surgery and had lesions 
larger than $5 \mathrm{~cm}$ in diameter or multiple lesions. Four patients with a solitary tumor smaller than $5 \mathrm{~cm}$ in diameter were treated in a single session because they refused the standard method. Patients with prothrombin activity less than $40 \%$ or a platelet count less than $40,000 / \mathrm{mm}^{3}$ were excluded. Diagnosis was based on results of sonographically guided fine-needle biopsy in 10 patients and on $\alpha$ fetoprotein levels that were greater than $200 \mathrm{ng} / \mathrm{ml}$ when imaging studies showed that the lesion was consistent with HCC in the others. Imaging showed that 10 patients had a single lesion ranging from 2.3 to $10.0 \mathrm{~cm}$ in diameter (mean, $5.7 \mathrm{~cm}$ ), and 10 patients had from two to five lesions each (for a total of 37 lesions) ranging from 0.9 to $8.0 \mathrm{~cm}$ in diameter (mean, $3.1 \mathrm{~cm}$ ). Eight of the patients with multiple lesions had already received standard treatment with $\mathrm{PEI}$; three of these patients had lesions that were recurrences due to incomplete treatment and the others had new lesions. $\alpha$-Fetoprotein levels were more than $200 \mathrm{ng} / \mathrm{ml}$ in 10 of the 20 patients with HCC.

Patients with metastases included six men and four women, 48 to 81 years old (mean, 63 years old). Patients were selected because they could not have or refused surgery. Diagnosis was based on results of sonographically guided fine-needle biopsy in four patients and on levels of carcinoembryonic antigen that were greater than 5 $\mathrm{ng} / \mathrm{ml}$ when imaging studies showed that the lesions were compatible with metastases and the lesions had not been present on the previous images. The primary tumor, resected in all cases, was of the colon or rectum in eight patients, of the breast in one patient, and of the gallbladder in one patient. Imaging studies showed that four patients had a single lesion ranging from 2.8 to $5.7 \mathrm{~cm}$ in diameter (mean, $4.7 \mathrm{~cm}$ ), five had from two to four lesions each (for a total of 15 lesions) ranging from 1.1 to $5.8 \mathrm{~cm}$ in diameter (mean, $3.2 \mathrm{~cm}$ ), and one had confluent lesions occupying $40 \%$ of the hepatic parenchyma. Levels of carcinoembryonic antigen were more than $5 \mathrm{ng} / \mathrm{ml}$ in all patients with metastases from the gastrointestinal tract.

In all patients, aspartate aminotransferase (AST), alanine aminotransferase (ALT), alkaline phosphatase, bilirubin, amylase, and hemoglobin levels, prothrombin time, platelet count, and RBCs and WBCs were evaluated before PEI.

\section{Technique}

We used a commercially available sonography scanner with a 3.5- $\mathrm{MHz}$ convex probe and guide attachment or with a 3.5-MHz linear probe that had an incorporated guide. Two types of needles were used to instill the ethanol: a 22-gauge spinal needle (Becton-
Dickinson, Rutherford, NJ) and a 21-gauge needle with closed conical tip and three terminal side-holes (Hakko, PEIT needle, Tokyo, Japan). As a rule, the spinal needle was used for lesions smaller than $3 \mathrm{~cm}$ or for small areas left unperfused by the multihole needle, and the other needle was used for the other lesions.

The procedure was performed with general anesthesia, endotracheal intubation, and mechanical ventilation. This kind of anesthesia is well suited for this procedure because it allows periods of controlled apnea or respiratory standstill with partial pulmonary inflation as requested. At the start of $\mathrm{PEI}, 20$ patients received fructose-1,6diphosphate (Esafosfina, Biomedica Foscama, Ferentino, Italy) 1000-1500 mg and glutathione-SH (TAD 600, Biomedica Foscama, Rome, Italy) $1200 \mathrm{mg}$ IV drip in $30 \mathrm{~min}$ with the aim of neutralizing the toxic systemic effects of injected alcohol by speeding up its metabolism. Blood alcohol level was measured immediately after treatment and $2 \mathrm{hr}$ and $24 \mathrm{hr}$ later. Depending on the site of the lesion, patients were placed supine or in a left lateral decubitus position. Patients received three to 22 (mean, 12) injections of sterile 95\% ethanol (SALF, Bergamo, Italy), depending on the size of the lesion and the mode of perfusion, until perfusion of the lesion was presumed to be complete, that is, until the entire lesion appeared to be more echogenic than the surrounding tissue at sonography (Fig. 1). Care was taken to inject the deepest portions of the lesion first, then the central and finally the superficial portions, in order to prevent superficial spillage of ethanol from masking the view for subsequent injections. Ethanol was injected slowly ( $1 \mathrm{ml}$ in 5-10 sec) and its diffusion was monitored with real-time sonography. As long as the ethanol was seen to disappear rapidly from the needle tip, injection was continued until ethanol pooled in the lesion.

\section{Follow-up Studies}

AST, ALT, alkaline phosphatase, bilirubin, amylase, and hemoglobin levels, prothrombin time, platelet count, and RBCs and WBCs were evaluated $2 \mathrm{hr}, 24 \mathrm{hr}, 48 \mathrm{hr}$, and 10 days after PEI. Levels of tumoral markers ( $\alpha$-fetoprotein and carcinoembryonic antigen) were measured and sonography and CT were performed 1 month after treatment. Long-term follow-up ranged from 6 to 18 months (mean, 10.5 months). Follow-up controls consisted of (1) sonography, measurement of levels of tumor markers, and liver function tests every 3 months and (2) unenhanced and contrast-enhanced CT every 6 months. In all patients, contiguous, $1-\mathrm{cm}$-thick sections were imaged. lopamidol (lopamiro 300 , Bracco, Milan, Italy) was administered as a $100-\mathrm{ml}$ bolus $(2-3 \mathrm{ml} / \mathrm{sec})$ followed by a rapid infusion of

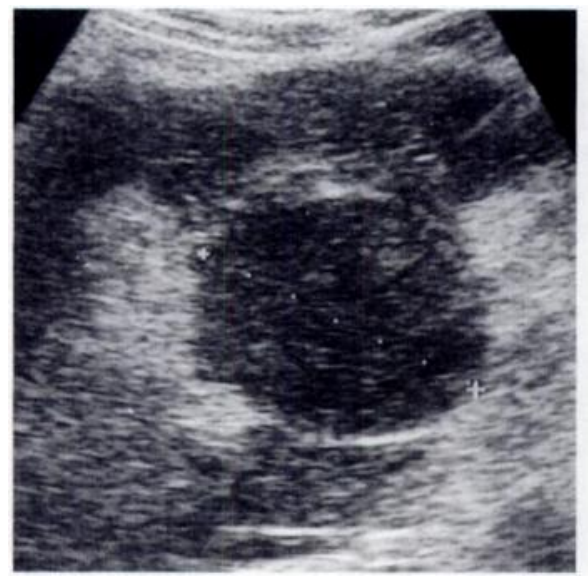

A

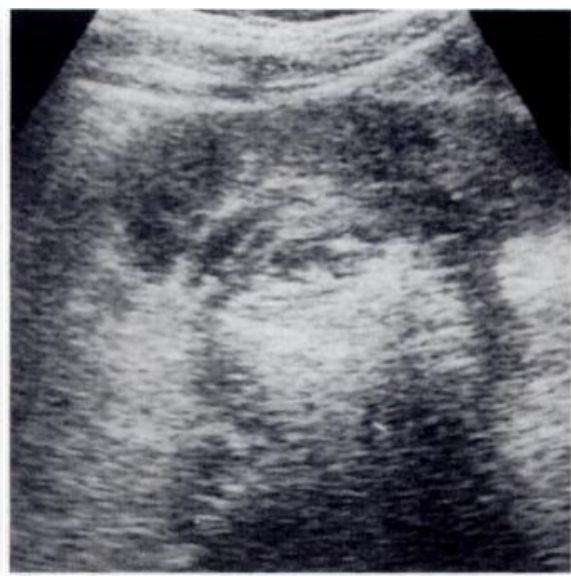

B
Fig. 1.-Single, encapsulated lesion of hepatocellular carcinoma in left lobe, $6.2 \mathrm{~cm}$ in diameter.

A, Transverse sonogram obtained before percutaneous injection of ethanol (PEI) shows lesion (cursors) of mixed echogenicity.

$B$, Sonogram obtained just after single-session PEI (80 $\mathrm{ml}$ in 16 injections) shows lesion homogeneously perfused by ethanol, without extravasation. 
50-100 $\mathrm{ml}$, depending on body weight. Scanning began $30 \mathrm{sec}$ after the start of contrast administration. Additional 5-mm-thick sections were imaged when more precise details were needed. We rated the response to treatment as complete when the CT scans showed no areas of enhancement within the lesion 1 month after treatment, when this finding was confirmed in the follow-up scans, and when the CT scans and sonograms showed no increase in tumor volume. We did not use the serologic measurements of tumor marker levels to assess response to treatment because those levels can return to normal when the scans do not show complete response (due to near-total necrosis of the tumor tissue) or decrease even when the scans do show complete response (due to the presence of intrahepatic or extrahepatic lesions that were not detected or were undetectable). We rated the response as partial when the CT scan showed more than $50 \%$ necrosis and the tumor marker levels had decreased.

\section{Results}

The total volume of alcohol administered per patient ranged from 6 to $165 \mathrm{ml}$ (mean, $57 \mathrm{ml}$ ). The smaller amount was given to a patient with a single $H C C 2.3 \mathrm{~cm}$ in diameter, and the greatest amount was given to a patient with confluent metastatic lesions occupying $40 \%$ of the hepatic parenchyma. Time required for the instillation of alcohol was 20$50 \mathrm{~min}$ (mean, $30 \mathrm{~min}$ ). The average hospital stay was 2.2 days for patients with metastases and 2.9 days for those with HCC. During the 3-4 days after treatment, 26 patients had tenderness in the right hypochondrium and 12 had hyperpyrexia $\left(38-38.5^{\circ} \mathrm{C}\right)$; hyperpyrexia developed in patients who received more than $50 \mathrm{ml}$ of alcohol.

Transient mild changes in the results of liver function tests, in particular an increase in transaminase levels, were observed in all but two cases. The highest level of AST was $868 \mathrm{U} / \mathrm{l}$ (normal, 5-35 U/I) and of ALT was $770 \mathrm{U} / \mathrm{l}$ (normal, 7-56 U/I). The increase in transaminase level was directly correlated with tumor volume and consequently with the quantity of ethanol injected. In all cases, the transaminase level returned to near the level before treatment in 10 days.

Blood alcohol levels immediately after treatment were 10 $229 \mathrm{mg} / 100 \mathrm{ml}$ (mean, $82 \mathrm{mg} / 100 \mathrm{ml}$ ) and $2 \mathrm{hr}$ later were 3-142 $\mathrm{mg} / 100 \mathrm{ml}$ (mean, $47 \mathrm{mg} / \mathrm{ml}$ ). In all patients, the values returned to normal within $24 \mathrm{hr}$. The time required for serum levels of ethanol to retum to normal was not appreciably different between patients who were treated with drugs during PEI and those who were not, but the former regained consciousness more easily and quickly. From the day after treatment, imaging examinations showed gas formation within the lesions in about $80 \%$ of patients, which was recognizable for 3-15 days.

Some complications occurred. Three patients with superficially located HCCs, without a cuff of normal hepatic parenchyma between the liver capsule and the lesion, had bleeding into the peritoneum. In all patients, the decrease in hemoglobin level was minimal $(2 \mathrm{~g} \%)$ and did not require transfusion. Two patients with HCC had necrosis of nonneoplastic tissue adjacent to the tumor with a marked increase in transaminase levels (AST, 1580 and $1341 \mathrm{U} / \mathrm{L} ;$; ALT, 2263 and $1819 \mathrm{U} / \mathrm{L}$ ); these patients had pain lasting 2-3 days that required analgesics (Fig. 2). In two patients with HCC, sonograms obtained 1 month after ethanol injection showed thrombosis of a segmental portal vein tributary to the tumor; this finding disappeared spontaneously 3 months after treatment.

\section{Follow-up of Hepatomas}

Seven of the 10 patients who had an encapsulated lesion had complete response, and three had about $90 \%$ necrosis (Figs. 3 and 4). One of the latter patients underwent two additional injections with standard PEI, after which response was complete. All complete responses observed after treatment were confirmed during follow-up. One patient with an encapsulated lesion $3.4 \mathrm{~cm}$ in diameter who showed complete response on imaging studies underwent liver transplantation 6 months later, which revealed $100 \%$ necrosis of the neoplastic tissue. In patients with infiltrating lesions, necrosis was extensive ( $70-90 \%)$, although it could not be evaluated precisely with imaging studies. Elevated $\alpha$-fetoprotein levels in the four patients with an encapsulated lesion returned to normal in all cases: from 217, 510, 2340, and $2870 \mathrm{ng} / \mathrm{ml}$ to less than $20 \mathrm{ng} / \mathrm{ml}$. Elevated $\alpha$-fetoprotein levels in the six patients with infiltrating lesions decreased in all cases: from 246, 320, 955, >3000, >3000, and 5640
Fig. 2.-Hepatic necrosis from extravasation of ethanol.

A, Contrast-enhanced CT scan shows low density of sixth hepatic segment, with gas formation in nonneoplastic parenchyma. This pattern, due to hepatic necrosis after extravasation of ethanol from a lesion in fitth segment, was accompanied by abdominal pain and a significant transient increase in transaminase level.

$B, C T$ scan obtained after 6 months of treatment shows well-defined area of low density (17 H).

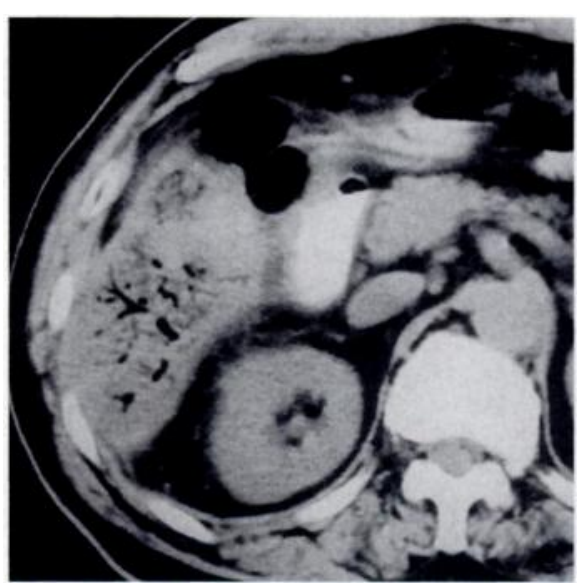

A

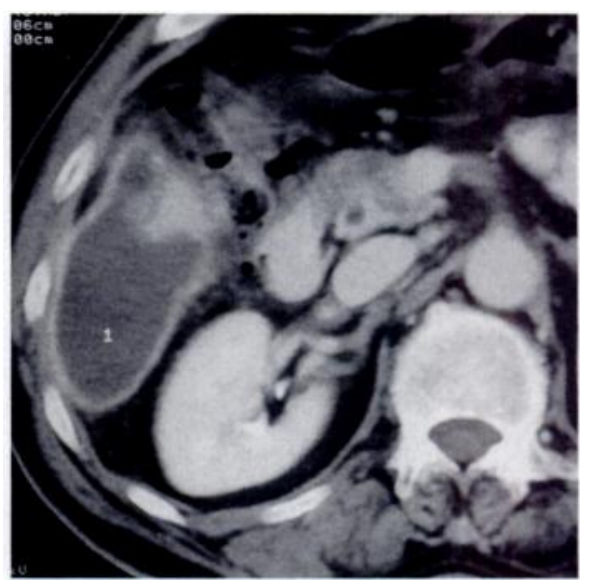

B 


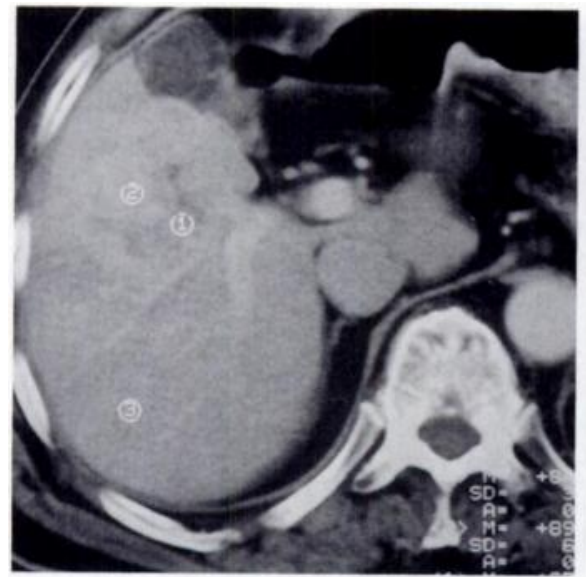

A

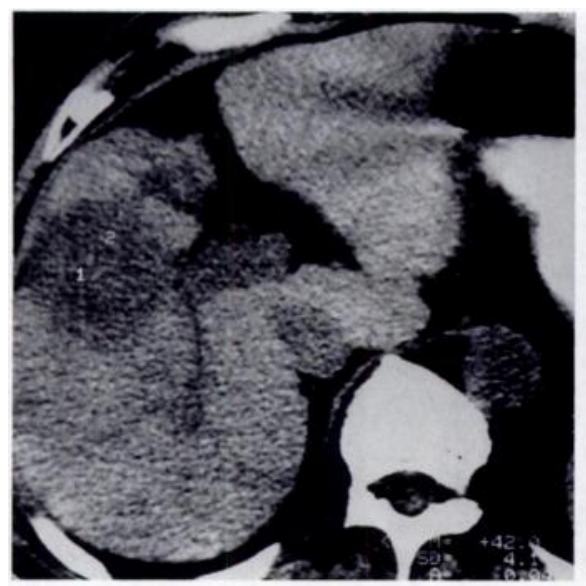

C

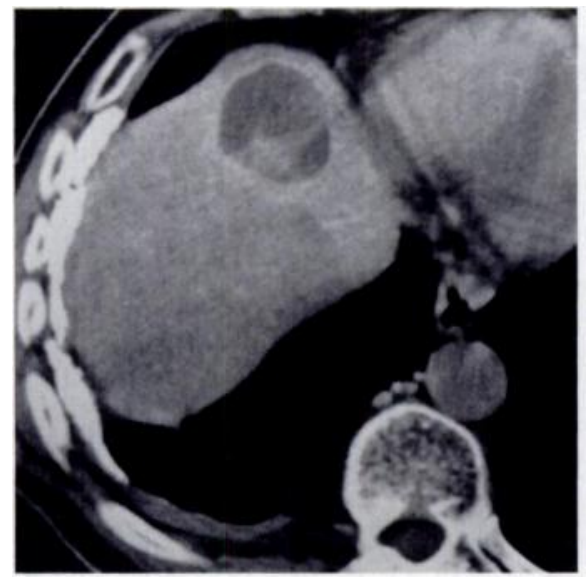

A

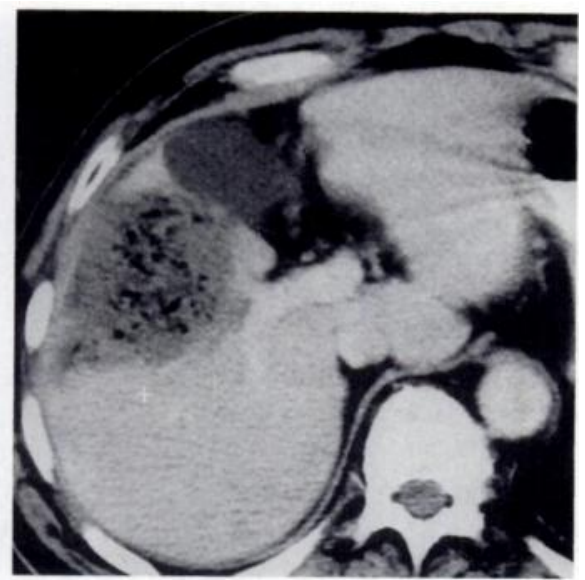

B

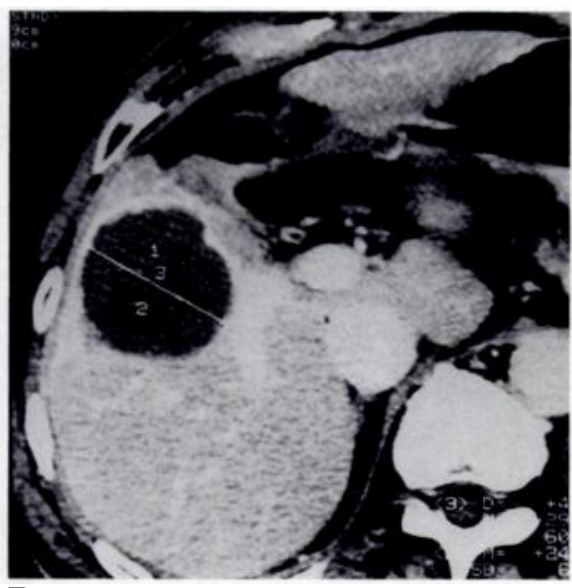

D

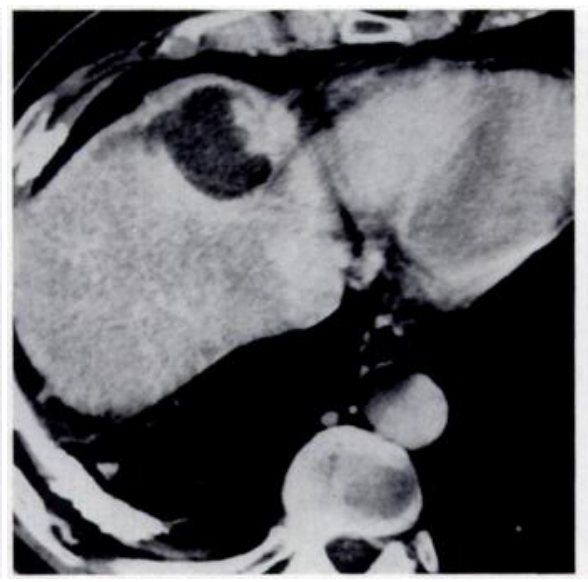

B
Fig. 3.-Single, encapsulated hepatocellular carcinoma, $5.6 \mathrm{~cm}$ in diameter.

A, CT scan obtained before treatment shows inhomogeneous area of high density $(69-87 \mathrm{H})$

$B, C T$ scan obtained 1 day after treatment (60 $\mathrm{ml}$ in 12 injections) shows gas formation within lesion.

$C$ and $D$, After 12 months, complete response is confirmed. Lesion shows no enhancement: density is same on unenhanced CT $\operatorname{scan}(C, 35$ and $42 \mathrm{H})$ and on contrast-enhanced $C T$ scan $(D, 24$ and $43 \mathrm{H})$. Level of $\alpha$-fetoprotein before percutaneous injection of ethanol was $2870 \mathrm{ng} / \mathrm{ml} ; 1$ month and 12 months after treatment, level had returned to normal (<20 $\mathbf{n g} / \mathbf{m l}$ ).

Fig. 4.-Single, encapsulated hepatocellular carcinoma, $4.5 \mathrm{~cm}$ in diameter.

A, Contrast-enhanced CT scan obtained before percutaneous injection of ethanol (PEI) shows lesion.

$B, C T$ scan obtained 1 month after single-session PEI (50 $\mathrm{ml}$ in 13 injections) shows partial response to treatment. Small area of high density that remains at periphery of lesion represents neoplastic tissue.

C, Sonogram shows same viable area (cursor), which was subsequently treated with two additional percutaneous injections of ethanol by standard technique, yielding a complete response.

$\mathrm{ng} / \mathrm{ml}$ to $79,60,396,260,568$, and $1500 \mathrm{ng} / \mathrm{ml}$, respectively. During follow-up, new lesions were detected in two patients 6 months after treatment and in one patient 3 months after treatment. Three months after treatment, one patient died of liver failure caused by obstructive jaundice due to hilar metastatic lymphadenopathy. 


\section{Follow-up of Metastases}

The response evaluated by imaging was partial, around $60-80 \%$, in all patients. No complications occurred. Levels of carcinoembryonic antigen, measured 1 month after treatment, decreased in all patients: from $13,30,70,83,156,188,226$, and $1396 \mathrm{ng} / \mathrm{ml}$ to $9,18,8,27,42,108,35$, and $560 \mathrm{ng} / \mathrm{ml}$, respectively. During follow-up, new hepatic lesions or local recurrences were detected in all patients. One patient died of his underlying disease 9 months after treatment.

\section{Discussion}

The rationale for the use of PEI in HCC is based on the following tenets: sonographic screening of patients with cirmosis allows detection of tumors that are relatively small and confined to the liver; the difference in density between the soft neoplastic tissue and the hard cirmotic tissue around it allows alcohol to diffuse easily throughout the tumor without extravasating into liver tissue; highly concentrated injection of alcohol causes intratumoral arterial thrombosis followed by tumoral ischemia; PEI spares liver tissue surrounding the HCC; and PEI is safer than surgery, which carries a higher risk of mortality and morbidity. In the treatment of small HCCs, PEI now has a well-defined role, with a 3-year survival rate similar to that for surgery [7].

Until recently, PEI has been used to treat only lesions that are less than $5 \mathrm{~cm}$ in diameter; it was thought that larger lesions would require an inordinate number of injections and hence an unduly long course of treatment, with no guarantee that the whole lesion would be treated. With the single-session technique, we aimed to expand the use of PEI to larger and more numerous lesions (avoiding at the same time some disadvantages such as pain and long treatment times). Although singlesession PEI started as a purely palliative technique, the results were unexpectedly good in encapsulated lesions, suggesting a more long-term response in these cases. In fact, complete response was obtained even with large tumors up to $8.2 \mathrm{~cm}$ in diameter, as confirmed at 1-year follow-up. In infiltrating HCC, the single-session technique enabled massive but not always complete necrosis with a transient decrease in $\alpha$-fetoprotein levels. For infiltrating or multicentric tumors, in which surgery is contraindicated, intraarterial therapy in some form is usually given. The amount of necrosis achievable with these methods is unpredictable, with a mean response of about $45 \%$ [8]. Considering the degree of necrosis obtained with the single-session technique in this study, we believe that this method could be offered as an alternative to intraarterial therapy. We now treat all patients with multiple lesions or single lesions greater than 5 $\mathrm{cm}$ in diameter with single-session PEI. Those with small solitary lesions are treated by the traditional approach to obviate general anesthesia.

The rationale for the use of PEI in hepatic metastases includes the assumption that destruction of the greatest possible quantity of neoplastic tissue should prolong survival. However, the role of PEI is not yet clear. Complete responses have been obtained only in patients with small endocrine metastases; in most other patients, with metastases from colon cancer greater than $3 \mathrm{~cm}$ in diameter, responses were almost always partial [6]. Considering the long time it takes to treat those patients, most of whom have multiple lesions, and the uncertainty of the outcome, our aim with the single-session technique was to make treatment more rapid, less painful, and therefore more acceptable. The response was only partial, with necrosis greater than $50 \%$ and only a transient decrease in levels of carcinoembryonic antigen. Necrosis was incomplete even in the smaller lesions, in contrast to previous reports with the standard PEI technique [6]. As metastases from colon cancer are often not encapsulated, alcohol penetrates the liver tissue, making further injections difficult. Because this problem is less apparent when the standard method is used, with its smaller quantity of ethanol injected, all our patients with inoperable metastases greater than $4 \mathrm{~cm}$ in diameter (whether single or multiple) receive the single-session treatment, while those with a single lesion less than $3 \mathrm{~cm}$ in diameter are still treated with the standard procedure.

Some complications occurred in patients with cirrhosis and HCC. Necrosis of nonneoplastic hepatic tissue, due to extravasation of ethanol, was not foreseeable but does not seem to have been a major problem for patients (the only complaint was pain). However, because liver function is transiently impaired in these patients, advanced cirrhosis might be a contraindication for the single-session technique. Peritoneal hemorrhage, which occurred in three patients with superficial lesions, did not require further treatment. Only one case of major peritoneal hemorrhage has been reported [4]. The bleeding that occurred in our patients was probably due to the sum of small amounts of bleeding from the numerous small injuries caused by needle insertion made on the liver surface. Chemical thrombosis of the segmental portal vein tributary to the lesion has sometimes been reported in the PEI series, but without related problems $[2,6]$. We were even more prepared for this to occur after the single-session technique because of the larger dose of alcohol, but as in the reported cases, the thrombosis disappeared spontaneously in our patients.

We conclude that single-session PEI is feasible, is acceptable to patients, and in some cases yields excellent longterm results. Nevertheless, the complications that occurred in some patients with cirrhosis prompt caution. More experience is needed before the indications for this procedure can be clearly defined.

\section{REFERENCES}

1. Livraghi T, Festi D, Monti F, Salmi A, Vettori C. US-guided percutaneous alcohol injection of small hepatic and abdominal tumors. Radiology 1986;161:309-312

2. Ebara M, Ohto M, Sugiura N, Okuda K, Kondo F, Kondo K. Percutaneous ethanol injection for the treatment of small hepatocellular carcinoma: study of 95 patients. Gastroenterol Hepatol 1990;5:616-626

3. Livraghi T, Vettori C. Percutaneous ethanol injection therapy of hepatoma Cardiovasc Intervent Radiol 1990;13:146-152

4. Shiina S, Tagawa K, Unuma T, et al. Percutaneous ethanol injection therapy of hepatocellular carcinoma: analysis of 77 patients. AJR 1990;155:12211226

5. Tanikawa K. Non-surgical treatment of hepatocellular carcinoma. Jpn J Gastroenterol Surg 1990;23:2492-2496

6. Livraghi T, Vettori C, Lazzaroni S. Liver metastases: results of percutaneous ethanol injection in 14 patients. Radiology 1991;179:709-712

7. Livraghi T, Bolondi L, Lazzaroni S, et al. Percutaneous ethanol injection in the treatment of hepatocellular carcinoma in cirthosis. Cancer 1992;68:926-929

8. Takayasu K, Shima Y, Muramatsu Y, et al. Hepatocellular carcinoma: treatment with intraarterial iodized oil with and without chemotherapeutic agents. Radiology 1987;162:345-351 